\title{
Opfer einer irgendwie gearteten Hirnaktivität?
}

Jürg Kesselring

\section{«Die Tragik solcher Behauptungen liegt darin, dass Kategorien vermischt werden, die man besser trennen sollte.»}

Korrespondenz:

Prof. Dr. med. Jürg Kesselring Chefarzt Klinik für Neurologie und Neurorehabilitation Rehabilitationszentrum CH-7317 Valens

j.kesselring[at]klinik-valens.ch
Mittlerweile scheint sich alles mit dem Präfix «Neuro» erneuern zu wollen. Ein lesenswertes Buch Neuroculture des Neurowissenschafters Ed Rolls [1] listet auf und diskutiert die Kapitel: Neuroscience, Neuroaffect, Neurosociality, Neuroreason, Neurophilosophy, Neuroaesthetics, Neuroethics, Neuropsychiatry, Neuroreligion, Neuropolitics. Wie der Ausdruck besagt, hängt alles an einem Faden (= neuron). Nun also auch die Theologie.

Dr. Knechts Beschreibung der empirischen Forschung zeigt dies anhand einschlägiger Forschungsergebnisse. Da kommt heraus, dass die Reizung gewisser Hirnareale zu Erlebnissen führen kann, die ohne solche Reizung auch schon beschrieben worden sind (z.B. als religiöse Erlebnisse), und die Tatsache, dass sie durch Reizung hervorgerufen werden können, wird als Indiz dafür gewertet, dass sie sozusagen unabhängig von der Person erfolgen, also nicht etwa subjektiv seien, wie der Erfahrene meint, sondern tatsächlich objektiv, also z.B. «nur» elektrische Reizmuster oder logische Folgezustände bestimmter

Gen- und Molekülkombinationen. Die Tragik solcher Behauptungen liegt darin, dass Kategorien vermischt werden, die man besser trennen sollte: Unsere eigenen menschlichen Erfahrungen, zu denen wohl auch das Religiöse gehört, sind nicht durch einzelne Molekülkonstellationen oder Reizstromstärken oder Magnetresonanzbilder in einem experimentell definierten Zeitpunkt ausreichend zu erklären. Erfahrungen haben immer auch eine Geschichte, die in der Messung einzelner Messparameter untergeht. Wundert es jemanden, dass auch Erfahrungen mit Gehirnfunktionen einhergehen? Dass auch sie sich im Laufe der Entwicklung herausgebildet haben?

Die Grundfrage, die wohl auch als Glaubensfrage aufgefasst werden kann, ist doch die, ob wir uns selbst so erleben, dass wir unsere Lebensfunktionen in einer natürlichen Umwelt und in einem persönlichen, sozialen Umfeld aktiv ausüben (ohne uns freilich immer aller Einzelheiten bewusst zu sein) oder ob wir uns nur passiv als Opfer einer irgendwie gearteten Hirnaktivität oder einem Determinismus aus der Umwelt ausgeliefert empfinden. «Genetisch bedingt» bedeutet ja nicht einfach unveränderbar: Das Beispiel Phenylketonurie, bei dem der genetische Defekt, der daraus resultierende Enzymmangel und das Krankheitsbild genau bekannt sind, zeigt, dass die Konsequenz dieser genetischen Determination durchaus vermieden werden kann, wenn nur schon auf den Nahrungsbestandteil des Phenylalanins verzichtet wird. Entscheidend ist es eben, die Interaktionen zu untersuchen und diese auf ein Ziel hin zu steuern. Viele Neuro-Artefakte entstehen dadurch, dass meist nur Einzelgehirne und oft auch nur diejenigen von Versuchstieren oder in Computersimulationen untersucht werden (weil dies technisch einfacher ist), wo doch in der Natur immer nur Interaktionen zwischen den lebendigen Trägern solcher Gehirne vorkommen, die ihre eigene Geschichte haben, die in einem natürlichen und sozialen Umfeld stetig neu angepasst wird. Hier lauert wieder die Gefahr von Kategorienfehlern, wenn aus solchen Artefakten direkt auf die Wirklichkeit und Wahrheit geschlossen wird und diese sich dann nachträglich den Versuchsbedingungen anpassen sollten. Gewiss mag es schwierig sein, sich den Indoktrinationen zu entziehen, besonders wenn diese beinhalten, dass man sich und anderen Leuten grundsätzlich keine Fragen stellen darf. Aber um sich zu bilden, hat man ja nicht nur ein Elternhaus oder eine Primarschulklasse oder einen einzelnen Religionsunterricht, in denen vielleicht solche Indoktrinationen vorgenommen worden sein mögen, sondern auch Freunde, Literatur, Reisemöglichkeiten, die auch andere, weitere Aspekte der Bildung ermöglichen. Das Abwägen der Einflüsse muss ohnehin immer durch den Einzelnen geschehen in Abhängigkeit von seiner Geschichte und im Hinblick auf seine Wünsche und Ziele. Solches Abwägen der Erfahrung, der Wahrnehmungsinhalte und der Handlungsmöglichkeiten im Kontext ist gewiss auch eine Hirnfunktion.

Die Welt ist ein Buch - und man kann - Gott sei Dank! - selbst entscheiden (wenn man lesen kann), ob man nur bei einer Seite ein Leben lang (+ evtl. noch eine Ewigkeit dazu) bleiben will. Poesie und Musik heilen die Wunden, die der Verstand schlägt, oder sonst kann man immer noch wieder einmal ein Blumenbeet betrachten oder nachts ins Freie treten und in den Himmel schauen.

\section{Literatur}

1 Rolls ET. Neuroculture. On the implications of brain science. Oxford University Press: 2012. 